\title{
Meditate to create: the impact of focused-attention and open-monitoring training on convergent and divergent thinking
}

\author{
Lorenza S. Colzato*, Ayca Ozturk and Bernhard Hommel \\ Institute for Psychological Research and Leiden Institute for Brain and Cognition, Leiden University, Leiden, Netherlands
}

\author{
Edited by: \\ Anna M. Borghi, University of \\ Bologna, Rome, Italy \\ Reviewed by: \\ Anna M Borghi, University of \\ Bologna, Rome, Italy \\ Thomas Kleinsorge, Leibniz Research \\ Centre for working environment and \\ Human Factors, Germany \\ *Correspondence: \\ Lorenza S. Colzato, Cognitive \\ Psychology Unit, Department of \\ Psychology, Leiden University, \\ Wassenaarseweg 52, 2333 AK \\ Leiden, Netherlands. \\ e-mail: colzato@fsw.leidenuniv.nl
}

\begin{abstract}
The practice of meditation has seen a tremendous increase in the western world since the 60s. Scientific interest in meditation has also significantly grown in the past years; however, so far, it has neglected the idea that different type of meditations may drive specific cognitive-control states. In this study we investigate the possible impact of meditation based on focused-attention (FA) and meditation based on open-monitoring (OM) on creativity tasks tapping into convergent and divergent thinking. We show that FA meditation and OM meditation exert specific effect on creativity. First, OM meditation induces a control state that promotes divergent thinking, a style of thinking that allows many new ideas of being generated. Second, FA meditation does not sustain convergent thinking, the process of generating one possible solution to a particular problem. We suggest that the enhancement of positive mood induced by meditating has boosted the effect in the first case and counteracted in the second case.
\end{abstract}

Keywords: meditation, creativity, divergent thinking, convergent thinking, open-monitoring, focused-attention

\section{INTRODUCTION}

The practice of meditation has seen a tremendous increase in the western world since the 60s (Murphy and Donavon, 1997). Scientific interest in meditation has also significantly grown in the past years (Cahn and Polich, 2006) and increasing evidence suggests the efficacy of meditation in health care and the field of stress management (Chiesa and Seretti, 2009) and some potency to enhance positive feelings (Chang et al., 2004) increase pain tolerance, and reduce anxiety (Wachholtz and Pargament, 2005). Thus, meditation seems to be an effective tool for enhancing general well-being.

In previous studies, meditation training has been shown to enhance some cognitive processes, such as the allocation of attentional resources in attention-demanding tasks (Brown et al., 1984a,b; Slagter et al., 2007). Less clear is the connection between meditation and creativity, to which the present study was devoted (see Horan, 2009, for a review). While some studies found evidence for a strong positive impact of meditation practice on creativity (Orme-Johnson and Granieri, 1977; Orme-Johnson et al., 1977; Ball, 1980), others found only a weak association or no effect at all (Cowger, 1974; Domino, 1977). However, the methodological diversity across these studies with regard to sample characteristics and type of meditation is considerable, which renders it questionable whether they were actually assessing the same construct and processes. Moreover, there is still no mechanistic model explaining how creative processes operate and how different type of meditations might affect these operations, which in view of the lack of conceptual clarity may not be surprising. To address this issue, we tried to avoid addressing meditation and creativity as a whole but, rather, focused on particular, relatively well-defined meditation techniques and specific subcomponents of creative performance.
More concretely, we investigated the impact of focused-attention (FA) meditation and open-monitoring (OM) meditation on creativity tasks tapping into convergent and divergent thinking.

Regarding meditation, FA and OM meditation represent the main techniques of Buddhist meditation practices (Lutz et al., 2008), even though many exercises represent mixtures of these two types (Cahn and Polich, 2006). In FA meditation, the individual focuses on a particular item, thought, or object. Everything else that might tend to attract attention, such as bodily sensations, environmental noise, or intrusive thoughts, is to be actively ignored by redirecting attention constantly back on the same focus point. In OM meditation, instead, the individual is open to perceive and observe any sensation or thought without focusing on a concept in the mind or a fixed item; therefore attention is flexible and unrestricted.

Regarding creativity, Guilford $(1950,1967)$ has distinguished between two main ingredients of most creative activities: divergent and convergent thinking - even though other processes may also contribute (Wallas, 1926). Divergent thinking is taken to represent a style of thinking that allows many new ideas being generated, in a context where more than one solution is correct. The probably best example is a brainstorming session, which has the aim of generating as many ideas on a particular issue as possible. Guilford's (1967) Alternate Uses Task (AUT) to assess the productivity of divergent thinking follows the same scenario: participants are presented with a particular object, such as a pen, and they are to generate as many possible uses of this object as possible. In contrast, convergent thinking is considered a process of generating one possible solution to a particular problem. It emphasizes speed and relies on high accuracy and logic. Mednick's (1962) Remote Associates Task (RAT) that aims to assess convergent thinking fits 
with this profile: participants are presented with three unrelated words, such as "time," "hair," and "stretch," and are to identify the common associate ("long"). Interestingly for our purposes, performance on the AUT and the RAT were found to be uncorrelated (Akbari Chermahini and Hommel, 2010) and differently affected by the same experimental manipulations (Hommel et al., submitted), which supports Guilford's (1967) suggestion that convergent and divergent thinking represent different components of human creativity.

Hommel (2012) and Hommel et al. (submitted) have argued that convergent and divergent thinking call for different cognitivecontrol states. Based on general considerations regarding the processes underlying human decision-making (for a review, see Bogacz, 2007) and on the possible impact of practice on these processes (Colzato et al., 2008), Hommel and colleagues suggested that two parameters may play a central role in generating creative acts. For one, people may exert strong or weak top-down control (in the sense of Duncan, 2001) on the process that is searching for the solution(s) of a given creative problem, such as an item in a creativity task. Strong top-down control focuses the search on very few or just one item that satisfies a number of well-defined criteria, whereas weak top-down control broadens the search space to activate many items that satisfy loosely defined criteria. For another, people may be able to implement a strong or weak degree of mutual inhibition between alternative representations, which again increases or decreases the competition for selection. From this perspective, divergent thinking (as assessed by the AUT) would be likely to require or benefit from a control state that provides a minimum of top-down control and local competition, so that the individual can easily and quickly "jump" from one thought to another in an only weakly guided fashion (Hommel, 2012; Hommel et al., submitted). In contrast, convergent thinking (as assessed by the RAT) would be likely to benefit from a strong top-down bias, which would heavily constrain and direct the search process, and from strong local competition (as only one solution can be (correct) $)^{1}$.

Colzato et al. (2008) have argued that practicing tasks and skills might establish chronic biases of cognitive-control toward states or state parameters that these tasks and skills require. In their own study, this argument was applied to bilinguals, who were assumed to acquire a bias toward relatively strong top-down control and local competition, so to stay within one language and not occasionally switching to another. Colzato et al. predicted that this bias might generalize to other, non-lingual tasks that benefit or suffer from the hypothesized cognitive-control bias. This prediction was confirmed by the observation that bilinguals perform more poorly

\footnotetext{
${ }^{1}$ One might object that some degree of memory search (and "jumping") is involved in both the AUT and the RAT because, in the RAT people need to frequently check candidate items against the three constraining concepts. Indeed, both tasks comprise of convergent components (as the search must be goal-directed) and divergent components (as even in the RAT people are likely to consider multiple answers), and it would be misleading to consider them as pure measures of convergent and divergent thinking, respectively (Hommel, 2012). Nevertheless, it is fair to say that the convergent component is more important in the RAT than in the AUT while for the divergent component the opposite holds. It is these relative differences that our hypotheses are based on, but we do not exclude that the two tasks are also sharing a couple of aspects. Importantly, this latter possibility works against (rather than for) our hypotheses and makes the empirical test rather conservative.
}

than monolinguals in a task that requires distributed-attention. Using the same theoretical framework, Colzato et al. (2010a) showed that practicing Calvinists, who can be assumed to have acquired a bias toward focused top-down control (Colzato et al., 2008), perform worse than well-matched atheists in a distributedattention task. Applying this theoretical framework to the practice of meditation, one would expect that particular types of meditation practice (OM vs. FA) establish particular biases toward particular cognitive-control states. Given its goal and characteristics, OM meditation would seem to call for rather weak and "allowing" top-down guidance (Lutz et al., 2008), which allows jumping from one thought to another. In contrast, FA meditation would seem to call for a strong degree of top-down control, which steers and efficiently constrains the search for the right thought or concept. If so, engaging in OM meditation should facilitate subsequent performance in tasks that require weak, "distributed" control (as presumably required by the AUT task) while engaging in FA meditation should be beneficial for subsequent performance in tasks requiring a more focused control style (as presumably required by the RAT task).

We investigated this hypothesis by having participants practicing OM- and FA-type meditation, in addition to a third visualization exercise that was considered as a baseline, and then performing a divergent thinking and a convergent thinking task. We expected that OM meditation practice would improve divergent thinking but not convergent thinking, while FA meditation practice should have the opposite effect. A complication that can be expected to work against this prediction is that any kind of practice remotely related to meditation or relaxation techniques are likely to improve mood (Chang et al., 2004), which again has been found to facilitate divergent thinking but not convergent thinking (Akbari Chermahini and Hommel, in press). This suggests that the benefits of OM meditation on divergent thinking might be easier to demonstrate than the benefits of FA meditation on convergent thinking. To check whether mood is affected by the two meditation techniques, and whether it is affected the same way, we also assessed perceived mood.

\section{MATERIALS AND METHODS PARTICIPANTS}

Nineteen healthy adults (13 females and 6 males) practitioners of both FA and OM meditation, (the average length of meditation practice in both FA and OM was about 2.2 years), aged $30-56$, served as participants and volunteered to the study without a financial reward, except a Leiden University coffee mug as gift. Informed consent was obtained from all participants after the nature of the study were explained to them. The protocol was approved by the local ethical committee (Leiden University, Faculty of Social and Behavioral Sciences).

\section{MEDITATION SESSIONS Focused-attention meditation}

Similarly to Samatha, which aim is to focus on a particular object, the participants use the natural breath as an anchor to focus attention at a particular point, i.e., parts of the body: "During the inhalation bring the attention to that particular area and exhale from there." The aim of instructing to focus the attention to the body part was to constantly revive the awareness and effort of the 
participant to sustain the intensity of the focus (and therefore the intended top-down control) throughout the whole session.

\section{Open-monitoring meditation}

As OM meditation we used an adapted version of the Transformational Breath ${ }^{\circledR}$ (TB) developed by Dr. Judith Kravitz in 1980 (Kravitz, 2002; de Jager, 2007). Breath was used as a vehicle to "set the mind free" and allow any thoughts, sensations, and emotions that arise. "Opening" or "being open" is a key concept that is encountered in the practice of TB (Kravitz, 1996, 2002; de Jager, 2007). The first goal is to open the breath, then open the mind to any occurring thought, sensation, or emotion and consequently expand the consciousness to a (spiritual) connection where one reaches clarity. During a session, as a thought occurs, most often accompanied by an emotion and/or a sensation in the body, the practitioner observes and acknowledges the experience without any judgment. A TB coach invites the practitioner to be open and instructs the meditator to observe rather than judge thoughts and emotions, leading to more readily accept all feelings and forms of emotions arising from moment to moment (Kravitz, 2002).

During a TB session, after guiding the practitioner into achieving and maintaining the full, connected breathing pattern, the coach uses certain verbal guidance, and affirmations. Affirmations are self-liberating statements of fundamental truths (Kravitz, 2002; de Jager, 2007). The practitioner is repeatedly instructed by the coach simply to notice and accept what comes up while continuing to breathe without pausing (Kravitz, 2002). Examples of affirmations used in this study are: "I am open"; "I let go"; "I open and expand my consciousness"; and "I accept myself as I am."

\section{Baseline session (BA)}

As baseline session we used a guided visualization exercise in which participants were asked to visualize certain household activities such as cooking and giving a dinner party. In order to prevent the attention to be fixed at one particular point or concept during this session, attention was being switched between (a) visualizing the activity, and (b) contemplating about the activity (e.g., with instructions such as "Think of who you would like to invite").

\section{PROCEDURE AND DESIGN}

The participant and the coach laid on two separate mats (at a distance of about $1 \mathrm{~m}$ ) on the floor; half sitting with the back against a back-jack. Eyes were closed in all three conditions. The same instructor, certified in Samatha, Mindfulness, and TB training, provided the instruction for all three sessions. Participants served in three 45 -min sessions separated by 10 days. In one session they performed under the supervision of a certified meditation coach the FA meditation ( $35 \mathrm{~min}$ ) and completed for $10 \mathrm{~min}$ ( $5 \mathrm{~min}$ each) a short version of the RAT (based on Mednick, 1962, and translated into Dutch; see Akbari Chermahini et al., in press) and the AUT (Guilford, 1967). In the other two sessions the method was the same except that participants performed the OM meditation and the baseline session and completed new items of the RAT and AUT. The order of these three types of sessions was counterbalanced across participants by means of a Latin square. The RAT and AUT were scored by two independent readers blinded to the experimental conditions.

\section{REMOTE ASSOCIATION TASK (CONVERGENT THINKING)}

In this task, participants are presented with three unrelated words (such as time, hair, and stretch) and are asked to find a common associate (long). Our Dutch version comprised of 30 items (Cronbach's alpha $=0.85$; see Akbari Chermahini et al., in press). In the three sessions, participants completed 10 different items.

\section{ALTERNATE USES TASK (DIVERGENT THINKING)}

In this task, participants were asked to list as many possible uses for six common household items (brick, shoe, newspaper, pen, towel, bottle). In each of the three sessions, participants completed two different items. The results can be scored in several ways with flexibility, the number of different categories used, being the most consistent and reliable (Akbari Chermahini and Hommel, 2010).

\section{Originality}

Each response is compared to the total amount of responses from all of the subjects. Responses that were given by only $5 \%$ of the group count as unusual ( 1 point) and responses given by only $1 \%$ of them count as unique ( 2 points).

\section{Fluency}

The total of all responses.

\section{Flexibility}

The number of different categories used.

\section{Elaboration}

The amount of detail, e.g., "a doorstop" counts 0, whereas "a door stop to prevent a door slamming shut in a strong wind" counts 2 ( 1 point for introducing door slamming and another for providing further detail about the wind).

\section{SUBJECTIVE MEASURES OF MOOD}

Perceived mood was assessed once (after all three session) by means of a visual analog scale ranging from 0 (mood did not increase at all) to 5 (mood increased very much).

\section{STATISTICAL ANALYSIS}

Mood scores and five measures (from the two tasks) were extracted for each participant: originality, fluency, flexibility, and elaboration scores from the AUT, the number of correct items from the RAT. All measures were analyzed separately by means of repeated-measures ANOVAs with Session (OM vs. FA vs. BA) as within-subjects factor and order of Session as covariate (in order to account for possible order effect). A significance level of $p<0.05$ was adopted for all tests.

\section{RESULTS}

Replicating earlier findings (Chang et al., 2004) mood increased after the OM and FA session, as compared to the BA session (see Table 1), $F(2,36)=59.53, p<0.0001, \mathrm{MSE}=0.460, \eta_{\mathrm{p}}^{2}=0.768$. Performance in the AUT and RAT was good and comparable to performance in other studies (e.g., Akbari Chermahini and Hommel, 2010); see Table 1.

As expected, all four scores of the AUT showed an advantage for the OM session over the FA and BA session. While this 


\begin{abstract}
Table 1 | Means and SD for originality, fluency, flexibility, and elaboration scores from the Alternate Uses Task (AUT), the number of correct items from the Remote Associates Task (RAT), and perceived mood ratings as a function of focused-attention training, Open-Monitoring training, and Baseline.
\end{abstract}

\begin{tabular}{lccc}
\hline Session & Focused-attention & Open-monitoring & Baseline \\
\hline AUT & & & \\
$\quad$ Elaboration & $0.1(0.5)$ & $0.4(1.1)$ & $0.1(0.3)$ \\
Fluency* $^{*}$ & $19.3(7.0)$ & $24.4(7.3)$ & $17.3(5.2)$ \\
Flexibility* & $5.4(2.7)$ & $7.7(4.4)$ & $3.5(2.8)$ \\
Originality* & $0.8(1.1)$ & $2.0(2.1)$ & $0.4(0.6)$ \\
RAT & $3.7(1.5)$ & $3.5(1.8)$ & $3.1(1.4)$ \\
Mood* $^{*}$ & $4.3(0.1)$ & $4.4(02)$ & $2.3(0.1)$ \\
\hline
\end{tabular}

${ }^{*} p<0.05$ (significant group difference).

advantage was reliable for flexibility, $F(2,36)=10.34, p<0.0001$, $\operatorname{MSE}=7.963, \eta_{\mathrm{p}}^{2}=0.365$, fluency, $F(2,36)=8.88, p<0.001$, MSE $=29.195, \eta_{\mathrm{p}}^{2}=0.330$, and originality, $F(2,36)=8.46$, $p<0.001, \mathrm{MSE}=1.726, \eta_{\mathrm{p}}^{2}=0.320$, it did not reach significance for elaboration, $F(2,36)=1.47, p=0.24, \mathrm{MSE}=0.514$, $\eta_{\mathrm{p}}^{2}=0.075$, see Table 1 .

Post hoc multiple comparisons tests revealed that during OM session practitioners showed greater flexibility, fluency and originality than in the BA session $(p=0.001 ; p=0.001 ; p=0.02)$ and the FA session ( $p=0.023 ; p=0.005 ; p=0.024)$, respectively.

No significant interaction of order of session on the four scores of the AUT was found, $F<1$.

In the RAT task participants did not report better (or worse) performance in the FA session than the $\mathrm{OM}$ and $\mathrm{BA}$ session, $F<1$, see Table 1. As in the case of the AUT task, no significant interaction of order of session on mean go RT was found, $F<1$.

\section{DISCUSSION}

The aim of our study to evaluate the possibility that different types of meditation (OM vs. FA) induce or bias people toward particular cognitive-control states. OM meditation was assumed to induce a relatively "distributed" cognitive-control state that is characterized by weak top-down biasing of information processing and weak local competition among alternative thoughts, while FA meditation was assumed to induce a relatively focused cognitive-control state characterized by strong top-down control and strong local competition. If so, OM meditation practice would be expected to facilitate divergent thinking, as assessed by the AUT, but not convergent thinking. And this is exactly what the data show: individuals excel in the AUT task after the OM meditation.

Our second prediction was that FA meditation practice should facilitate convergent thinking, as assessed by the RAT, but not divergent thinking. Even though RAT performance was best after FA meditation, this increase was far from significant. However, the mood scores show that both types of meditation practice elevated mood in comparable ways. Given that elevated mood facilitates divergent, rather than convergent thinking and may even interfere with the latter (Akbari Chermahini and Hommel, in press), it is possible that meditation practice affected convergent thinking in two opposite ways: the focused character of the meditation might have improved convergent thinking performance while the relaxing aspect of the procedure might have hampered it. However, at this point this is still a speculation that calls for further research, perhaps using more extended practice.

Our hypotheses are motivated by the idea that particular types of meditation might establish or prime a particular cognitivecontrol state, which then primes or interferes with the control states needed to perform a convergent- or divergent-thinking task. A different, but not necessarily incompatible interpretation might be considered in terms of transfer-appropriate practice. For instance, it might be that practicing a particular type of meditation is associated with the acquisition or practice of particular cognitive operations, which are useful for particular thinking task but not others. Whether one considers this interpretation as different from our control state interpretation depends on how one conceives of what is a control state and what is a cognitive operation. From a functional viewpoint, one might argue that a control interpretation locates the effect at a different processing level than operation learning - with the latter representing particular information transformation rules but the former the implementation of these rules. From a neural perspective, however, the difference between the interchanges may be negligible: if one considers a control state nothing but the current wiring and activation pattern of the neural network, it is evident that different wirings and activation patterns will lead to different kinds of information transformation. In other words, the differentiation between control states and the rules these states imply may make more conceptual than neural sense.

Given that our participants were practitioners of both FA and OM meditation, we considered a within-subject the most appropriate option. However, it needs to be mentioned that this design choice has the drawback that we cannot entirely exclude that self-selection and demand characteristics have biased our results. Future studies might include an originally naive control group practicing FA or OM meditation for some time, similarly to Lutz et al. (2004), which would allow testing whether self-selected practitioners differ systematically from "experimental" practitioners. Moreover, it seems important to replicate our results using other types of OM meditation than Mindfulness and FA meditation, such as the traditional Samatha. It would also be interesting to explore possible commonalities with other more or less ritual practices with and without religious backgrounds. In previous studies we have found that religious belief systems, like Calvinism, Catholicism, and Judaism, affect cognitive-control parameters in rather specific ways (Hommel and Colzato, 2010). In particular, practicing a particular religion seems to bias the preferred values of cognitive-control parameters toward a range that is likely to generate behavior that is approved by one's social environment (Colzato et al., 2008, 2010a). Similar findings were obtained with non-religious factors like sexual orientation (Colzato et al., 2010b), which suggests that it is not religion per se but the selective reward of particular behavioral outcomes - and of the cognitivecontrol parameters producing these outcomes - that matter. From that perspective, mediation may be seen as one of many cultural practices that systematically shape the way people control their cognitive processes.

In any case, at least for divergent thinking we were able to show that meditation has a specific impact. This suggests that not all types of meditation have the same effect, which 
might explain why previous studies failed to provide unequivocal evidence for positive effects of meditation on creativity. Importantly, it also suggests that the benefits of OM meditation go beyond mere relaxation and receiving attention from a supervisor, which participants also received in the other two conditions. Apparently, OM practice restructures cognitive processing to a degree that is robust and general enough to affect performance in another, logically unrelated task. We suggest that this kind of practice reduces the degree of top-down control and local competition and, thus, leads to a broader distribution of potential resources. This establishes, or biases the individual toward a cognitive-control state that is less focused and "exclusive," which facilitates jumping from one thought to

\section{REFERENCES}

Akbari Chermahini, S., Hickendorff, M., and Hommel, B. (in press). Development and validity of a Dutch version of the Remote Associate Task: an item response theory approach. Think. Skills Creativity. doi: 10.1016/j.tsc.2012.02.003. [Epub ahead of print].

Akbari Chermahini, S., and Hommel, B. (2010). The (b)link between creativity and dopamine: spontaneous eye blink rates predict and dissociate divergent and convergent thinking. Cognition 115, 458-465.

Akbari Chermahini, S., and Hommel, B. (in press). Creative mood swings: divergent and convergent thinking affect mood in opposite ways. Psychol. Res. doi: 10.1007/s00426-0110358z. [Epub ahead of print].

Ball, O. E. (1980). The Effect of TM and the TM-Sidhi Program on Verbal and Figural Creativity (TTCT), Auditory Creativity (S and I), and Hemispheric Dominance (SOLAT). Unpublished doctoral dissertation, University of Georgia.

Bogacz, R. (2007). Optimal decisionmaking theories: linking neurobiology with behavior. Trends Cogn. Sci. (Regul. Ed.) 11, 118-125.

Brown, D., Forte, M., and Dysart, M. (1984a). Differences in visual sensitivity among mindfulness meditators and non-meditators. Percept. Mot. Skills 58, 727-733.

Brown, D., Forte, M., and Dysart, M. (1984b). Visual sensitivity and mindfulness meditation. Percept. Mot. Skills 58, 775-784.

Cahn, B. R., and Polich, J. (2006). Meditation states and traits: EEG, ERP, and neuroimaging studies. Psychol. Bull. 132, 180-211.

Chang, V. Y., Palesh, O., Cladwell, R., Glasgow, N., Abramson, M., Luskin, F., Gill, M., Burke, A., and Koopman, C. (2004). The effects of a mindfulness-based stress reduction program on stress, mindfulness and self-efficacy, and positive states of mind. Stress Health 20, 141-147.
Chiesa, A., and Seretti, A. (2009). Mindfulness-based stress reduction for stress management in healthy people: a review and meta-analysis. J. Altern. Complement. Med. 15, 593-600.

Colzato, L. S., van Beest, I., van den Wildenberg, W. P. M., Scorolli, C., Dorchin, S., Meiran, N., Borghi, A. M., and Hommel, B. (2010a). God: do I have your attention? Cognition 117, 87-94.

Colzato, L. S., van Hooidonk, L., van den Wildenberg, W. P. M., Harinck, F., and Hommel, B. (2010b). Sexual orientation biases attentional control: a possible gaydar mechanism. Front. Psychol. 1:13. doi:10.3389/fpsyg.2010.00013

Colzato, L. S., van den Wildenberg, W., and Hommel, B. (2008). Losing the big picture: how religion may control visual attention. PLOS ONE 3, e3679. doi:10.1371/journal.pone. 0003679

Cowger, E. L. (1974). The effects of meditation (zazen) upon selected dimensions of personality development. Diss. Abstr. Int. 34(Pt 1), 4734.

de Jager, M. (2007). Een reis naar onafhankelijkheid met de kracht van ademen. Steenbergen: Inside-Out.

Domino, G. (1977). Transcendental meditation and creativity: an empirical investigation. J. Appl. Psychol. 62, 358-362.

Duncan, J. (2001). An adaptive coding model of neural function in prefrontal cortex. Nat. Rev. Neurosci. 2, 820-829.

Guilford, J. P. (1950). Creativity. Am. Psychol. 5, 444-454.

Guilford, J. P. (1967). The Nature of Human Intelligence. New York: McGraw-Hill.

Hommel, B. (2012). "Convergent and divergent operations in cognitive search," in Cognitive Search: Evolution, Algorithms, and the Brain. Strüngmann Forum Reports, Vol. 9, eds P. M. Todd, T. T. Hills, and T. W. Robbins (Cambridge, MA: MIT Press), 215-230.

another - as required in divergent thinking. This consideration fits with the observation of Slagter et al. (2007) that OM meditation leads to better performance in a distributed-attention task and reinforces the view that meditation practice can have a lasting and generalizable impact on human cognition (Lutz et al., 2008).

\section{ACKNOWLEDGMENTS}

The research of L. S. Colzato is supported by a Veni grant of the NWO (Netherlands Organization for Scientific Research). We thank Nico van Zon, Marco, and Jacquelien de Jager for their enthusiasm and invaluable assistance in recruiting and testing the participants of this study.

Hommel, B., and Colzato, L. S. (2010). Religion as a control guide: on the impact of religion on cognition. Zygon J. Relig. Sci. 45, 596-604.

Horan, R. (2009). The neuropsychological connection between creativity and meditation. Creat. Res. J. 21, 199-222.

Kravitz, J. (1996). Personal Transformation Seminar: Manual. Tilton: Transformational Breath Foundation, International Headquarters.

Kravitz, J. (2002). Breathe in, Laugh Loudly: The Joy of Transformational Breathing, Revised Edn. Center Sandwich, NH: Free Breath Press.

Lutz, A., Greischar, L., Rawlings, N. B., Ricard, M., and Davidson, R. J. (2004). Long-term meditators selfinduce high-amplitude synchrony during mental practice. Proc. Natl. Acad. Sci. U.S.A. 101, 16369-16373.

Lutz, A., Slagter, H. A., Dunne, J. D., and Davidson, R. J. (2008). Attention regulation and monitoring in meditation. Trends Cogn. Sci. (Regul. Ed.) 12, 163-169.

Mednick, S. (1962). The associative basis of creative problem solving process. Psychol. Rev. 69, 200-232.

Murphy, M., and Donavon, S. (1997). The Physical and Psychological Effects of Meditation: A Review of Contemporary Research with a Comprehensive Bibliography 1931-1996. Sausalito, CA: Institute of Noetic Sciences.

Orme-Johnson, D. W., Clements, G., Haynes, C. T., and Badaoui, K. (1977). "Higher states of consciousness: EEG coherence, creativity, and experiences of the sidhis," in Scientific research on Maharishi's Transcendental Meditation and TM-Sidh Program, Collected Papers, Vol. 1, eds D. W. Orme-Johnson and J. T. Farrow (New York: MERU Press), 705-712.

Orme-Johnson, D. W., and Granieri, B. (1977). "The effects of the age of enlightenment governor training courses on field independence, creativity, intelligence, and behavioral flexibility," in Scientific Research on Maharishi's Transcendental Meditation and TM-Sidhi Program, Collected Papers, Vol. 1, eds D. W. Orme-Johnson and J. T. Farrow (New York: MERU Press), 713-718.

Slagter, H. A., Lutz, A., Greischar, L. L., Francis, A. D., Nieuwenhuis, S., Davis, J. M., and Davidson, R. J. (2007). Mental training affects distribution of limited brain resources. PLoS Biol. 5, 138. doi:10.1371/journal.pbio. 0050138

Wachholtz, A. B., and Pargament, K. I. (2005). Is spirituality a critical ingredient of meditation? Comparing the effects of spiritual meditation, secular meditation, and relaxation on spiritual, psychological, cardiac, and pain outcomes. J. Behav. Med. 28, 369-384.

Wallas, G. (1926). The Art of Thought. New York: Harcourt Brace.

Conflict of Interest Statement: The authors declare that the research was conducted in the absence of any commercial or financial relationships that could be construed as a potential conflict of interest.

Received: 27 January 2012; accepted: 30 March 2012; published online: 18 April 2012.

Citation: Colzato LS, Ozturk A and Hommel B (2012) Meditate to create: the impact of focused-attention and open-monitoring training on convergent and divergent thinking. Front. Psychology 3:116. doi: 10.3389/fpsyg.2012.00116 This article was submitted to Frontiers in Cognition, a specialty of Frontiers in Psychology.

Copyright (c) 2012 Colzato, Ozturk and Hommel. This is an open-access article distributed under the terms of the Creative Commons Attribution Non Commercial License, which permits noncommercial use, distribution, and reproduction in other forums, provided the original authors and source are credited. 